\title{
CFD analysis and experimental investigations towards optimizing the parameters of Ranque-Hilsch vortex tube
}

\author{
Upendra Behera ${ }^{\text {a }}$, P.J. Paul ${ }^{\text {a }}$, S. Kasthurirengan ${ }^{\text {b }}$, R. Karunanithi ${ }^{\text {, }}$, \\ S.N. Ram ${ }^{b}$, K. Dinesh ${ }^{b}$, S. Jacob ${ }^{b, *}$ \\ a Department of Aerospace Engineering, Indian Institute of Science, Bangalore 560 012, India \\ b Centre for Cryogenic Technology, Indian Institute of Science, Bangalore 560 012, India
}

\begin{abstract}
Computational fluid dynamics (CFD) and experimental studies are conducted towards the optimization of the Ranque-Hilsch vortex tubes. Different types of nozzle profiles and number of nozzles are evaluated by CFD analysis. The swirl velocity, axial velocity and radial velocity components as well as the flow patterns including secondary circulation flow have been evaluated. The optimum cold end diameter $\left(d_{\mathrm{c}}\right)$ and the length to diameter $(L / D)$ ratios and optimum parameters for obtaining the maximum hot gas temperature and minimum cold gas temperature are obtained through CFD analysis and validated through experiments. The coefficient of performance (COP) of the vortex tube as a heat engine and as a refrigerator has been calculated.
\end{abstract}

Keywords: Ranque-Hilsch vortex tube; CFD simulation; Experimental validation; Design parameters; Thermal performance

\section{Introduction}

Ranque-Hilsch vortex tube having, relatively simple geometry, no moving mechanical parts and no need for absolute sealing element, was invented by Ranque [1] demonstrating the effect of temperature separation of gases. Later, Hilsch [2] described this effect in detail. Intensive experimental and analytical studies of Ranque-Hilsch effect began since then and continue even today. According to these studies when a vortex tube is injected with compressed gas through tangential nozzles into its scroll chamber, a strong cir-

\footnotetext{
${ }^{*}$ Corresponding author. Tel./fax: +918023601612.

E-mail address: jacob@ccf.iisc.ernet.in (S. Jacob).
}

cular flow field is established. This vortex in the inlet area causes pressure distribution of the flow in radial direction. As a result a free vortex is produced as the peripheral warm stream and a forced vortex as the inner cold stream. The flow pattern is schematically shown in Fig. 1. The tangential velocity of the fluid element is related to the distance $r$ from the axis of rotation as, $v \propto r$, for forced vortex or quasi-solid rotation and $v \propto 1 / r$, for free vortex or potential vortex. The variation of tangential velocity along the radius for such a case in a vortex tube representing forced region and free region is shown in Fig. 2.

Many investigators, Hilsch [2], Deissler and Perlmutter [3], Kurosaka et al. [4], Ahlborn et al. [5], Cockerill [6], Gutsol [7], have suggested various theories to explain the Ranque effect. Ahlborn et al. [14] have postulated a 


\begin{tabular}{|c|c|c|c|}
\hline \multicolumn{4}{|c|}{ Nomenclature } \\
\hline$A$ & area $\left(\mathrm{m}^{2}\right)$ & $C_{\varepsilon i}$ & coefficients $(i=1,2,3,4)$ used in Eq. (4) \\
\hline CFD & computational fluid dynamics & $\Delta T_{\mathrm{ch}}$ & temperature difference between cold and hot \\
\hline $\mathrm{COP}$ & coefficient of performance & & end \\
\hline$D$ & diameter of vortex tube $(\mathrm{mm})$ & $\Delta T_{\mathrm{ih}}$ & temperature difference between inlet and hot \\
\hline$d_{\mathrm{c}}$ & cold end diameter $(\mathrm{mm})$ & & end \\
\hline$h$ & enthalpy $\left(\mathrm{kJ} \mathrm{kg}^{-1} \mathrm{~K}^{-1}\right)$ & $\Delta T_{\mathrm{hi}}$ & temperature difference between hot end and \\
\hline he & heat engine & & inlet \\
\hline$k$ & turbulence kinetic energy $\left(\mathrm{m}^{2} \mathrm{~s}^{-2}\right)$ & & \\
\hline$L$ & length of vortex tube $(\mathrm{m})$ & \multicolumn{2}{|c|}{ Greek symbols } \\
\hline$m$ & mass flow rate $\left(\mathrm{kg} \mathrm{s}^{-1}\right)$ & $\alpha$ & cold gas fraction \\
\hline$p$ & piezometric pressure $(\mathrm{Pa})$ & $\beta$ & coefficient used in Eq. (4) \\
\hline$R$ & radius of vortex tube (mm) & $\varepsilon$ & turbulence dissipation rate $\left(\mathrm{m}^{2} \mathrm{~s}^{-3}\right)$ \\
\hline re & refrigerator & $\mu$ & dynamic viscosity $\left(\mathrm{kg} \mathrm{m}^{-1} \mathrm{~s}^{-1}\right)$ \\
\hline$r$ & radial distance from axis & $\mu_{\mathrm{t}}$ & turbulent viscosity $\left(\mathrm{kg} \mathrm{m}^{-1} \mathrm{~s}^{-1}\right)$ \\
\hline$S_{m}$ & mass source & $\eta_{0}$ & coefficient used in Eq. (4) \\
\hline$S_{i}$ & momentum source components & $\rho$ & density $\left(\mathrm{kg} \mathrm{m}^{-3}\right)$ \\
\hline$T$ & temperature $(\mathrm{K})$ & $\sigma$ & stress $\left(\mathrm{N} \mathrm{m}^{-2}\right)$ \\
\hline$t$ & time $(\mathrm{s})$ & $\tau$ & shear stress $\left(\mathrm{N} \mathrm{m}^{-2}\right)$ \\
\hline$u$ & swirl velocity $\left(\mathrm{m} \mathrm{s}^{-1}\right)$ & $\tau_{i j}$ & stress tensor components \\
\hline$u_{i}$ & absolute fluid velocity component in direc- & \multirow{2}{*}{\multicolumn{2}{|c|}{ Subscripts }} \\
\hline & tion $x_{i}$ & & \\
\hline$V_{0}$ & maximum swirl velocity $\left(\mathrm{m} \mathrm{s}^{-1}\right)$ & c & cold gas \\
\hline$v$ & radial velocity $\left(\mathrm{m} \mathrm{s}^{-1}\right)$ & $\mathrm{h}$ & hot gas \\
\hline$w$ & axial velocity $\left(\mathrm{m} \mathrm{s}^{-1}\right)$ & $\mathrm{i}$ & inlet in vortex tube \\
\hline$x_{i}$ & Cartesian coordinate $(i=1,2,3)$ & $\mathrm{r}$ & constant reference value \\
\hline$z$ & axial length from nozzle cross section & & \\
\hline
\end{tabular}

theory of temperature separation based on heat pump mechanism enabled by secondary circulation flow in vortex tubes. However till today no exact theory has come up to explain the phenomenon satisfactorily. Thus much of the design and development of vortex tubes have been based on empirical correlations leaving much scope for optimization of critical parameters.

Computational fluid dynamics techniques have revolutionized engineering design in several important areas, notably in analysis of fluid flow technology. CFD can also be used as a minimal adequate tool for design of engineering components. A careful scanning of various numerical investigations on the mech-

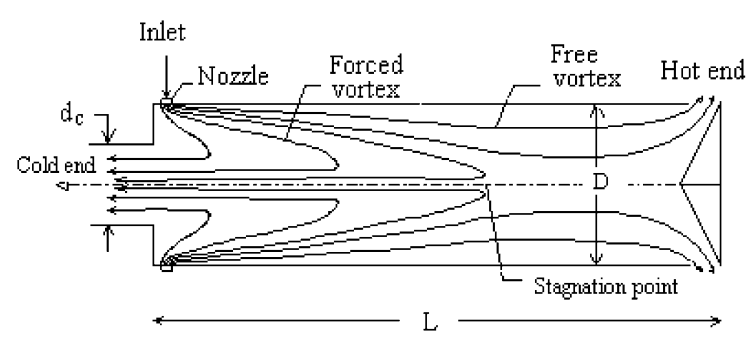

Fig. 1. Schematic flow pattern of Ranque-Hilsch tube. anism of thermal separation in vortex tubes indicate that barring a few [10], no serious attempts have been made to use CFD techniques to simulate the flow patterns of vortex tubes.

In the present study, a detailed analysis of various parameters of the vortex tube has been carried out

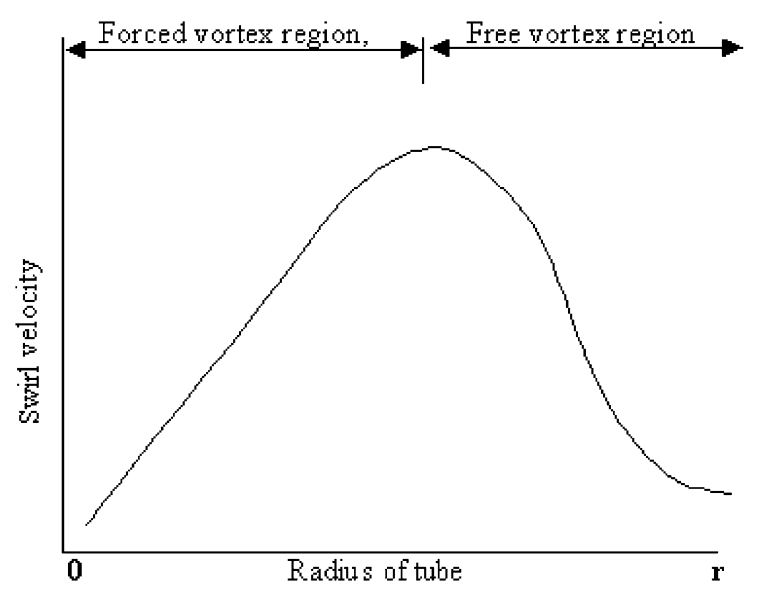

Fig. 2. Schematic velocity distribution in Ranque-Hilsch tube. 
through CFD techniques to simulate the phenomenon of flow pattern, thermal separation, pressure gradient etc. so that they are comparable with the experimental results. The paper reports also its utility as a tool for optimal design of vortex tube towards the optimization of number of nozzles, nozzle profiles, cold end diameter, length to diameter ratio, cold and hot gas fractions and comparison of the experimental results with corresponding CFD analysis.

\section{Numerical modeling of vortex tube}

The numerical modeling of the vortex tube has been carried with the code system of Star-CD. This programme allows calculations for incompressible, compressible and turbulent flow. The mass and momentum conservation equations per unit volume with respect to time (Star-CD Methodology and User Guide [11]) solved by Star-CD for general incompressible and compressible fluid flow are given by

$\frac{\partial}{\partial t}(\rho)+\frac{\partial}{\partial x_{j}}\left(\rho \tilde{u}_{j}\right)=S_{m}$

$\frac{\partial}{\partial t}\left(\rho u_{i}\right)+\frac{\partial}{\partial x_{j}}\left(\rho \tilde{u}_{j} u_{i}-\tau_{i j}\right)=-\frac{\partial p}{\partial x_{i}}+S_{i}$

where $\tilde{u}_{j}=u_{j}-u_{c j}$, relative velocity between fluid and local (moving) coordinate frame that moves with velocity $u_{c j}$.

The specialization of the above equations to a particular class of flow involves:

- application of ensemble or time averaging if the flow is turbulent;

- specification of a constitutive relation connecting the components of the stress tensor $\tau_{i j}$ to the velocity gradients;

- specifications of the 'source', $S_{i}$, which represents the sum of the body and other external forces, if present.

The scope of numerical model was restricted through few assumptions like steady state, symmetrical and compressible flows where the density itself could be estimated using the equation of state with the calculated temperature and pressure values.

\subsection{Turbulence model}

Flow in the vortex tube is highly turbulent. Star-CD employs mathematical models of turbulence to determine the Reynolds stresses and turbulent scalar fluxes. The steady state assumption and practical considerations indicate that a turbulence model must be employed to represent its effects. As the Reynolds number is very high, 'Renormalization Group' (RNG) version of $k-\varepsilon$ model has been used as this can be employed for 'law of the wall' functions.

The equations involved in $\mathrm{RNG} k-\varepsilon$ model for turbulence energy and turbulence dissipation rate are given as

$$
\begin{gathered}
\frac{\partial}{\partial t}(\rho k)+\frac{\partial}{\partial x_{j}}\left(\rho \tilde{u}_{j} k-\frac{\mu_{\mathrm{eff}}}{\sigma_{k}} \frac{\partial k}{\partial x_{j}}\right) \\
=\mu_{\mathrm{t}}\left(P+P_{\mathrm{B}}\right)-\rho \varepsilon-\frac{2}{3}\left(\mu_{\mathrm{t}} \frac{\partial u_{i}}{\partial x_{i}}+\rho k\right) \frac{\partial u_{i}}{\partial x_{i}} \\
\frac{\partial}{\partial t}(\rho \varepsilon)+\frac{\partial}{\partial x_{j}}\left(\rho \tilde{u}_{j} \varepsilon-\frac{\mu_{\mathrm{eff}}}{\sigma_{\varepsilon}} \frac{\partial \varepsilon}{\partial x_{j}}\right) \\
=C_{\varepsilon 1} \frac{\varepsilon}{k}\left[\mu_{\mathrm{t}} P-\frac{2}{3}\left(\mu_{\mathrm{t}} \frac{\partial u_{i}}{\partial x_{i}}+\rho k\right) \frac{\partial u_{i}}{\partial x_{i}}\right]+C_{\varepsilon} \frac{\varepsilon}{k} \mu_{\mathrm{t}} P_{\mathrm{B}} \\
-C_{\varepsilon 2} \rho \frac{\varepsilon^{2}}{k}+C_{\varepsilon 4} \rho \varepsilon \frac{\partial u_{i}}{\partial x_{i}}-\frac{C_{\mu} \eta^{3}\left(1-\eta / \eta_{0}\right)}{1+\beta \varepsilon^{2}} \frac{\rho \eta^{2}}{k}
\end{gathered}
$$

where $C_{\varepsilon 1}, C_{\varepsilon 2}, C_{\varepsilon 3}$ and $C_{\varepsilon 4}$ are given by $1.42,1.68,0.0$ or 1.42 (if $P_{\mathrm{B}}>0$ and 0 otherwise) and -0.387 respectively.

$\mu_{\text {eff }}=\mu+\mu_{\mathrm{t}}$

$P \equiv 2 S_{i j} \frac{\partial u_{i}}{\partial x_{j}}, \quad P_{\mathrm{B}}=-\frac{g_{i}}{\sigma_{h}} \frac{1}{\rho} \frac{\partial \rho}{\partial x_{i}}, \quad \eta \equiv S k / \varepsilon$,

$S \equiv\left(2 s_{i j} s_{i j}\right)^{1 / 2}, \quad \eta_{0}=4.38, \quad \beta=0.01$

\section{Physical modeling of vortex tube}

The vortex tube flow was investigated by modeling the $12 \mathrm{~mm}$ diameter vortex tubes used for the experimental programme with the Star-CD code. The meshing and analysis of vortex tubes have been carried out in a P4, $2.4 \mathrm{GHz}$ and $1 \mathrm{~GB}$ RAM machine with refinement of meshes near the critical regions such as nozzle inlet areas, hot end exit area and at wall boundary.

The analysis is carried out to arrive at the optimum number of nozzles, nozzle profile, cold end diameter $\left(d_{\mathrm{c}}\right)$, length to diameter $(L / D)$ ratio, cold and hot gas fractions. The diameter of vortex tube, $D=12 \mathrm{~mm}$ and ratio of nozzle inlet area to vortex tube area $A_{\mathrm{i}} / A=0.07$ are kept nearly constant in the analysis. The $L / D$ ratio assumed for the studies ranged from 10 to 35 .

\subsection{Boundary conditions for analysis}

The pressure and temperature data obtained from the experiments are supplied as input for the analysis. The boundary conditions given to simulate the vortex tube phenomenon at different regions are as follows. 
- Stagnation boundary condition to the inlet region of the vortex tube (at inlet of nozzle) with total pressure of $0.5422 \mathrm{MPa}$ (absolute) and total temperature of $300 \mathrm{~K}$.

- Pressure boundary condition to cold end region of vortex tube with pressure of $0.136 \mathrm{MPa}$ (absolute).

- Pressure boundary condition to the hot end region of vortex tube with pressure varying so as to vary the mass fraction at the hot end to obtain the optimum value.

The temperatures at hot and cold ends are given zero gradients in calculation and the mass flow rate at the outlet of nozzle is constant and the flow is compressible. As the flow inside vortex tube is axisymmetric, a part of sector is taken for analysis giving cyclic boundary condition. The three-dimensional model showing the boundary regions used in calculation is represented in Fig. 3(a) and (b). The reference pressure $p_{\mathrm{r}}=0.136 \mathrm{MPa}$ and reference temperature $T_{\mathrm{r}}=293 \mathrm{~K}$ are specified. The tube walls are considered to be adiabatic and no slip conditions are used. For the numerical solution of the equation for compressible flow, a variant of well-known SIMPLE algorithm method is used.

\subsection{Grid dependence study}

To eliminate the errors due to coarseness of grid, analysis has been carried out for different average unit cell volumes in a vortex tube of $L / D=10$. The variation of key parameters such as total temperature difference and maximum swirl velocity are shown in Fig. 4(a) and (b) for different unit cell volumes. It can be seen that not much advantage in reducing the unit cell volume size below $0.003 \mathrm{~mm}^{3}$ which corresponds to 0.75 million cells for the configuration studied.

\section{Velocity and temperature profiles in vortex tube from CFD analysis}

For a specific vortex tube of diameter $D=12 \mathrm{~mm}$, $L / D=20$, cold end diameter $d_{\mathrm{c}}=7 \mathrm{~mm}$ with six numbers of convergent nozzles the following analysis is made.

\subsection{Velocity components and flow pattern}

It has been known [6] that experimental determination of velocity components is rather difficult in vortex tube, because the measuring probes can considerably
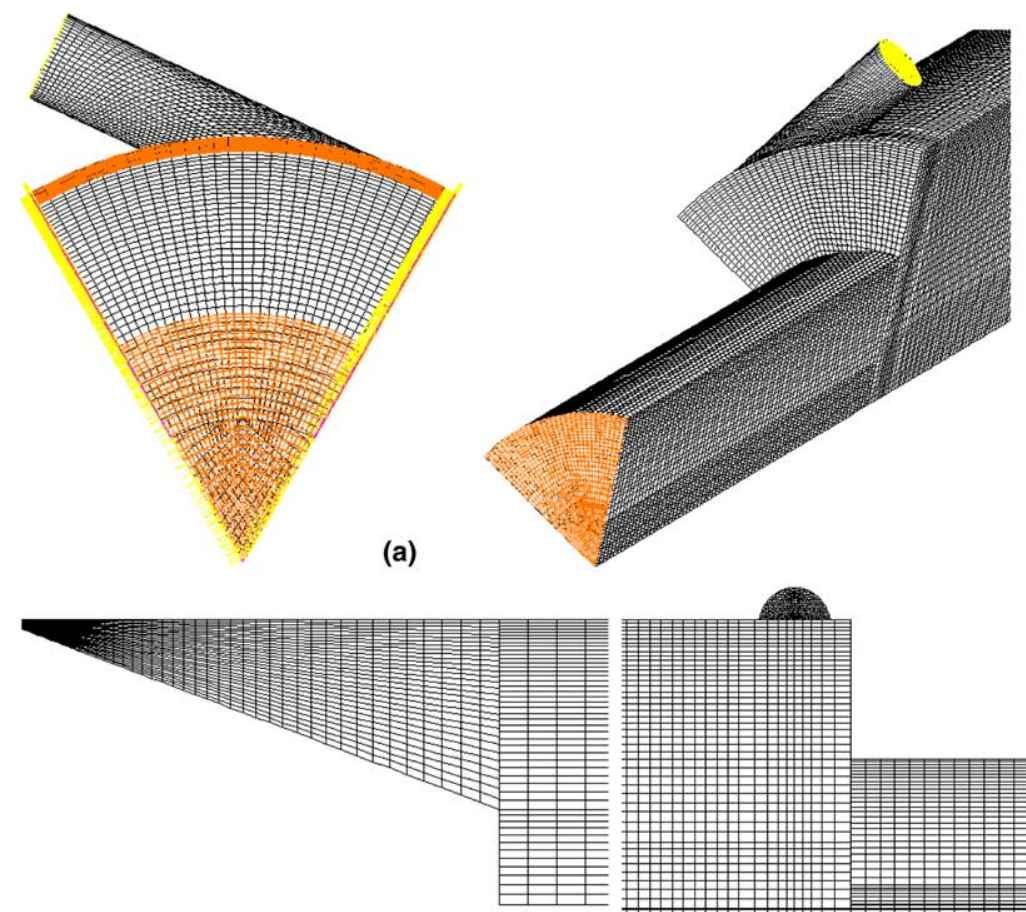

(b)

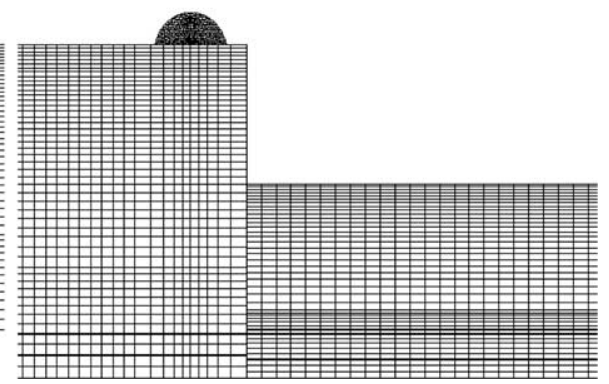

Fig. 3. (a) Three-dimensional model of vortex tube in sector; (b) the hot end and cold end of vortex tube in sector provided with refinement in mesh. 


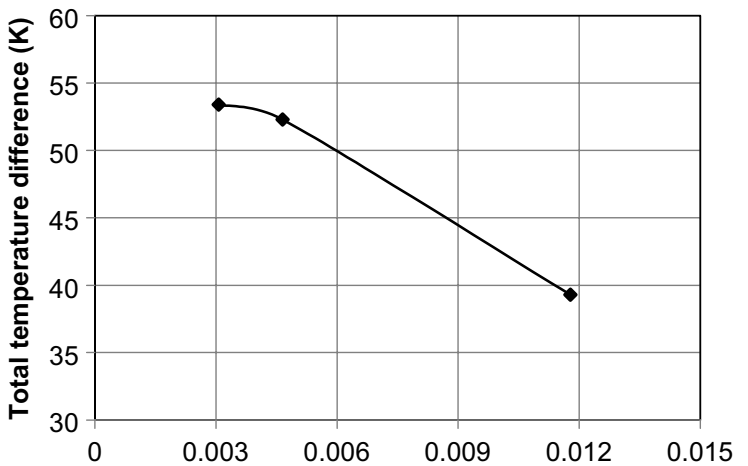

(a)

Average unit cell volume $\left(\mathrm{mm}^{3}\right)$

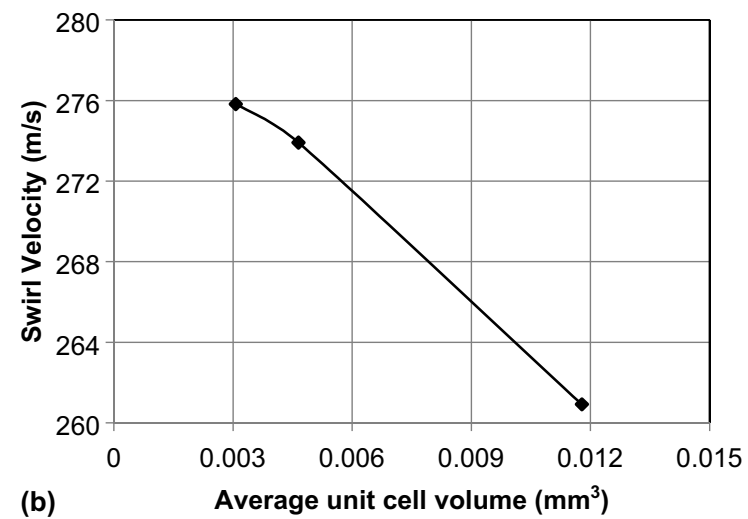

Fig. 4. Grid size dependence study on: (a) total temperature difference at different average unit cell volume; (b) maximum swirl velocity at different average unit cell volume.

alter the flow pattern. Even using a $1.6 \mathrm{~mm}$ diameter Pitot tube for a $25.4 \mathrm{~mm}$ diameter vortex tube, $8 \%$ of vortex tube cross section is blocked [13]. Therefore determination of the velocity components from CFD assumes great significance. The radial profiles of velocity components swirl, radial and axial are shown in Fig. 5(a)-(c) and the flow patterns at specific sectional lengths in Fig. 5(d). Comparing the velocity components, it is observed that swirl velocity ( $u$-component) has the highest value. It has a value almost equal to inlet tangential flow $V_{0}$ in the nozzle inlet zone, which rapidly decreases in amplitude towards the hot end discharge. The radial profile of the swirl flow indicates a free vortex near the wall but predominantly forced vortex especially at inlet section, which is in conformity with the observations of Kurosaka [4], Gutsol [7]. The small diameter of the vortex tube $(12 \mathrm{~mm})$ also leads to a predominantly forced vortex flow pattern.

The axial flow velocity ( $w$-component) shows a maximum value $w / V_{0} \approx-0.365$ at the tube axis near the inlet zone. The axial flow profile shows that the flow reversal of the forced vortex core flow takes place at about $8 \mathrm{~mm}$ diameter and the core flow fills up the cold flow outlet area $(7 \mathrm{~mm}$ diameter) completely. Stagnation point can be established from the velocity profile along the tube length at the point where axial velocity cease to have a negative value.

The radial velocity ( $v$-component) is significantly low in magnitude compared to the $u$ and $w$ components. It has a negative component at the inlet zone at radius $\approx 0.3 R$ towards the tube axis and a positive component upto the tube wall. This indicates possibilities of energy transfer in the radial plane.

The above velocity profiles obtained at different $z / L$ sections of vortex tube are in good conformity with those shown by Gutsol [7].

The experimental observation of flow pattern in metallic vortex tube is rather difficult, while CFD analysis can provide better understanding of this. The flow pattern obtained by CFD analysis for sectional lengths of $0.05 \mathrm{~m}$ at specific $z / L$ location is shown in Fig. 5(d). The core and peripheral flow can be clearly seen at $z / L$ sections at 0.0375 and 0.46 but at $z / L=0.95$ shows only the presence of peripheral flow towards the hot end. Thus CFD analysis can locate the stagnation point at appropriate $z / L$ location of the vortex tube.

\subsection{Temperature distribution}

The total temperature from CFD analysis plotted as contour plot in Fig. 6. It shows that peripheral flow is warm and core flow is cold relative to inlet temperature for a hot gas flow fraction of about $28 \%$, giving maximum hot gas temperature of $353.7 \mathrm{~K}$ and minimum cold gas temperature of $266.2 \mathrm{~K}$. For experiments conducted with nearly same conditions of hot gas flow fraction, the maximum hot gas temperature obtained is $\approx 360 \mathrm{~K}$ and minimum cold gas temperature $\approx 281 \mathrm{~K}$. Thus there is fair agreement in the total temperatures obtained through experiment and by CFD simulations.

\section{CFD analysis towards optimization of parameters}

\subsection{Nozzle profile and number of nozzles}

Earlier, investigators [9] had to carry out laborious fabrications and experimental programme to deduce the optimum nozzle profiles and evaluate their performances. CFD analysis can minimize these difficulties.

CFD analysis has been carried out on different types and number of nozzles to arrive at the optimum profile of the inlet nozzle and number of nozzle(s) for enhanced swirl generation. In order to provide uniform basis for comparison of results and to ensure symmetric conditions, the analysis have been done for the entire cross section and not for $60^{\circ}$ sector that is possible for six 

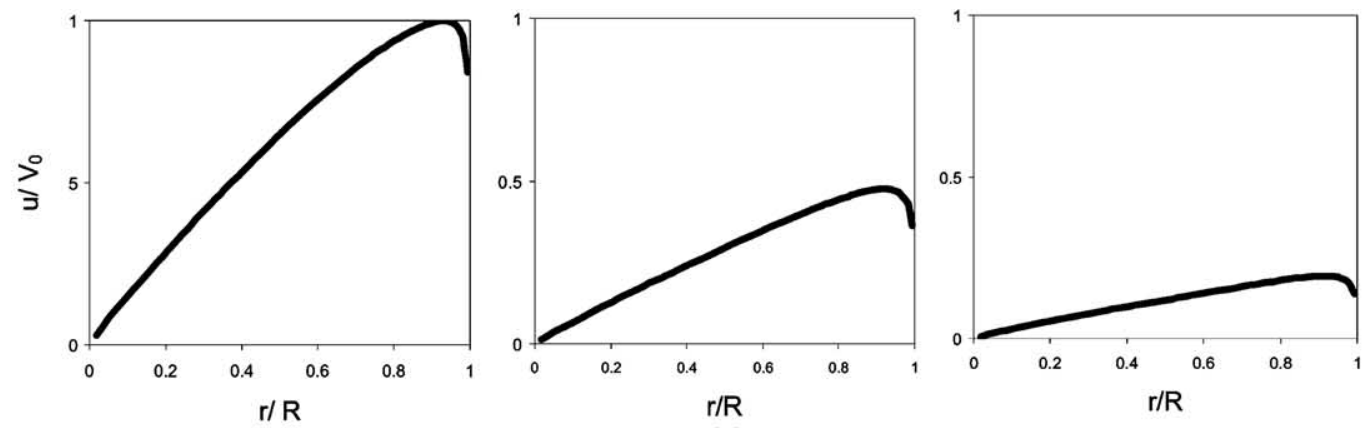

(a)
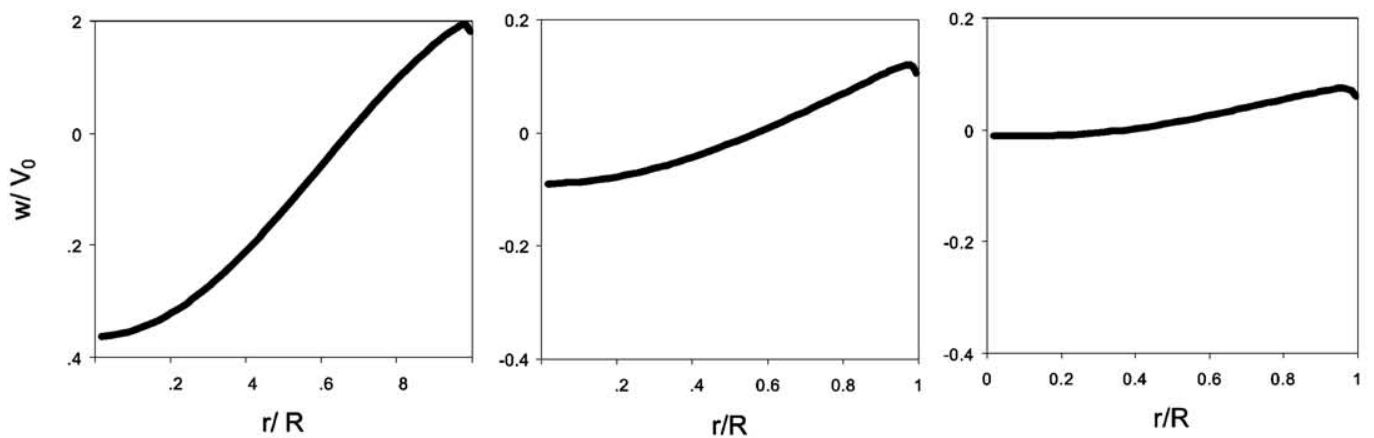

(b)
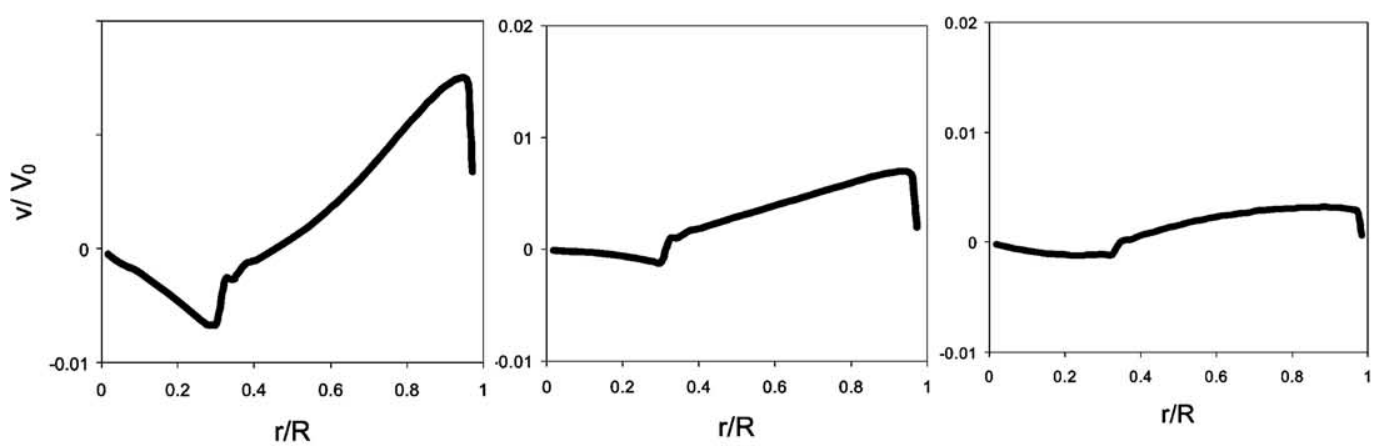

(c)
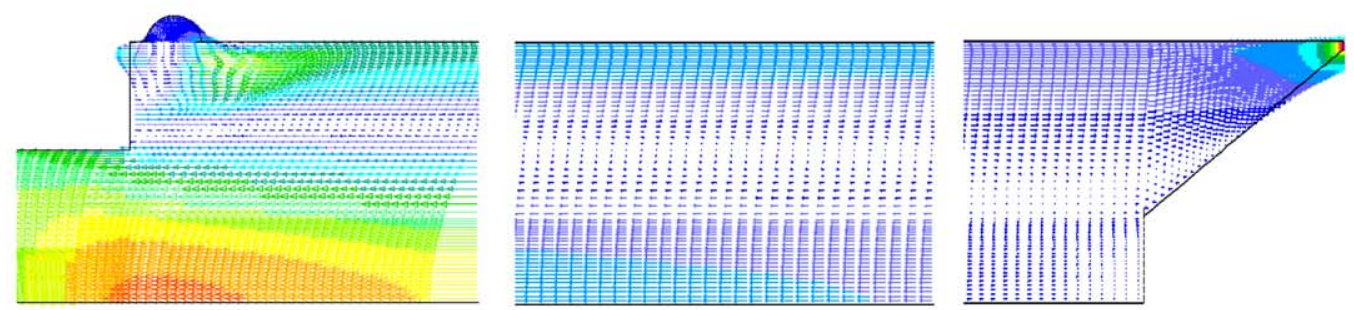

(d)

Fig. 5. (a) Radial profiles of swirl velocity ( $u$-component); (b) axial velocity ( $w$-component); (c) radial velocity ( $v$-component) at $z / L=0.0375,0.46,0.95$; (d) flow pattern for $0.05 \mathrm{~m}$ length sections at $z / L=0.0375,0.46,0.95$.

numbers of nozzles or $180^{\circ}$ sector that is possible for two numbers of nozzles. This may yield a lesser value of total temperature difference compared to sector analysis. However for comparison of nozzle profiles and number of nozzles, this approach is adequate. The geometric dimensions, boundary conditions and mass flow are kept nearly constant for all nozzle profiles in the analysis. 


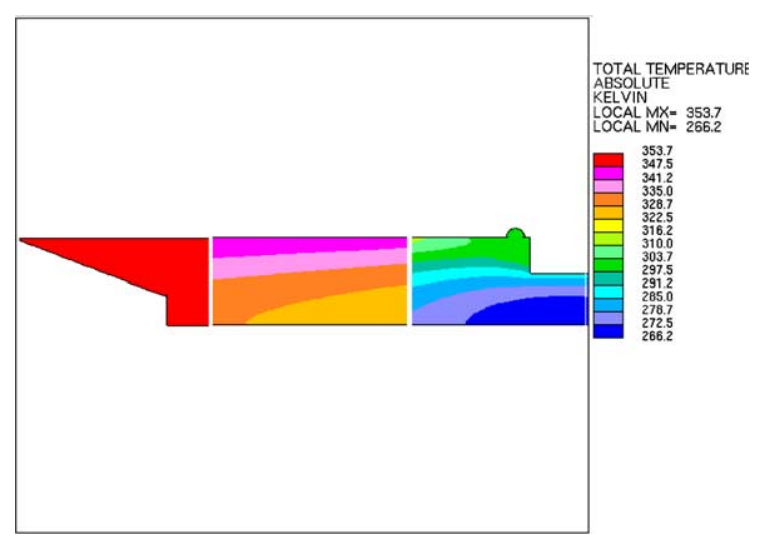

Fig. 6. Temperature distribution in axial direction of vortex tube in sections.

The swirl pattern in the vortex generator for different nozzle profiles are given in Fig. 7(a)-(e) and the result of analysis is given in Table 1. The performance of swirl generators can be characterized by two important factors, namely the magnitude of swirl velocity and radial symmetry of flow.

The flow pattern obtained for two numbers of convergent nozzles is oval in shape though the swirl velocity is highest. In this case the non-radial symmetry of the flow results in less total temperature difference. In the case of six convergent nozzles, radial symmetry of flow is obtained along with good swirl velocity, which has resulted in the highest total temperature difference compared to all the nozzle profiles. This is in conformity with the studies as reported by Gutsol [7], which mention that not less than four tangential nozzles are necessary to attain proper radial symmetry of flow. The swirl velocity attained for circular and rectangular helical single nozzles are significantly less than for conical nozzles and hence they give lower total temperature difference. Therefore a swirl generator with six numbers of convergent nozzles is selected for further design and analysis of vortex tubes.

\subsection{Cold end orifice diameter}

The temperature difference $\Delta T_{\mathrm{ch}}$ obtained experimentally for $L / D=10$ at specific hot gas fractions and at different cold end diameters of 5,6,7, and $7.5 \mathrm{~mm}$ and CFD simulation for 6 and $7 \mathrm{~mm}$ are shown in Fig. $8(\mathrm{a})$. It can be seen that maximum temperature difference of $53.4 \mathrm{~K}$ (at $15.75 \%$ of hot gas flow) is obtained from CFD analysis and about $62.9 \mathrm{~K}$ (at $13.51 \%$ of hot gas flow) is obtained from experiments at $d_{\mathrm{c}}=7 \mathrm{~mm}$. Thus CFD analysis is in reasonable agreement with experimental results.

From the analysis and experiments it has been observed that vortex tube can be operated in such a way that it can be used to produce maximum hot gas temperature and/or to produce minimum cold gas temperature. The CFD analysis and experimental results are plotted in Fig. 8(b) and (c) showing hot gas temperature and cold gas temperature at different hot gas fractions for specific cold end diameters at $L / D$ ratio of 10 . Fig. 8(d) and (e) shows the experimental data of maximum hot and minimum cold gas temperatures obtained for different cold end diameters and $L / D$ ratios. The optimum value of cold end diameter $d_{\mathrm{c}}$ to get maximum hot gas temperature is $7 \mathrm{~mm}$ where as to get minimum cold gas temperature $d_{\mathrm{c}}$ is $6 \mathrm{~mm}$, for all the values of $L / D$ greater than 10 . These values of cold end diameter fairly match with values reported by Takahama [12] and the correlation $d_{\mathrm{c}} / D=0.350+0.313 \alpha$ as given by Merkulov [8].

This study confirms that CFD modeling carried out in this work is capable of selecting the correct $d_{\mathrm{c}}$ value for a vortex tube, without resorting to the empirical correlations as a design guide or a laborious experimental programme.

\subsection{Length to diameter ratio}

Initial CFD analysis for specific $L / D$ ratio of 10 showed that the swirl intensity extends beyond the length of hot end exit of the tube and maximum $\Delta T_{\mathrm{ch}}$ to be $53.4 \mathrm{~K}$. Further investigations were carried out at different $L / D$ ratios in the range of $10-30$ to find the length of tube such that the stagnation point is within the tube. They led to the conclusion that increase in the length of tube enhances the temperature separation upto the condition that stagnation point is within the length of tube. The corresponding $L / D$ ratio is in the range of $20-30$.

Experiments were carried out for different $L / D$ ratios of 10 to 35. Fig. 9 shows the experimental and CFD analysis data on temperature difference $\Delta T_{\text {ch }}$ for different $L / D$ ratios. It can be noted that the peak value in $\Delta T_{\text {ch }}$ is obtained experimentally for $L / D$ ratio of 30 . The studies highlight that CFD has reasonable accuracy in predicting an optimum $L / D$ ratio and is an adequate tool for the design of the vortex tube.

\subsection{Hot gas fraction}

The influence of hot gas fraction on the hot gas temperature and cold gas temperature from CFD analysis is shown in Fig. 8(b) and (c) for vortex tube of $L / D=$ 10 . The results indicates that maximum hot gas temperature is obtained for lower mass fraction at the hot end and similarly lowest cold gas temperature is obtained at lower mass fraction at cold end (higher mass fraction at hot end).

Fig. 10(a) and (b) represents the experimental data of hot gas and cold gas temperatures at different hot gas 


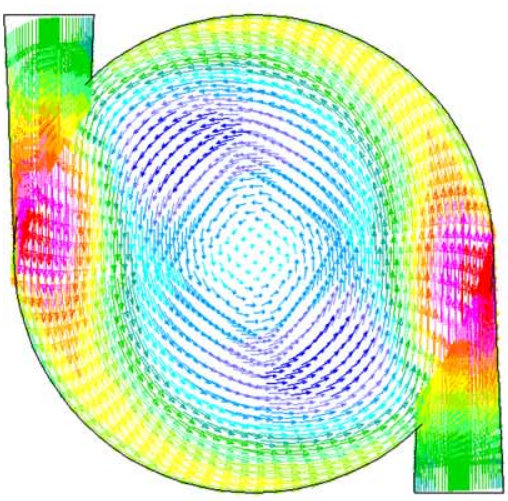

(a)

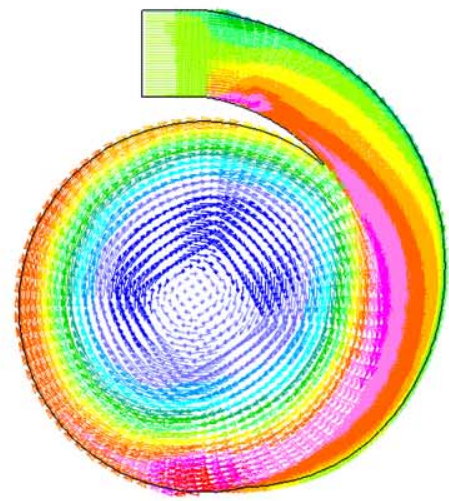

(c)

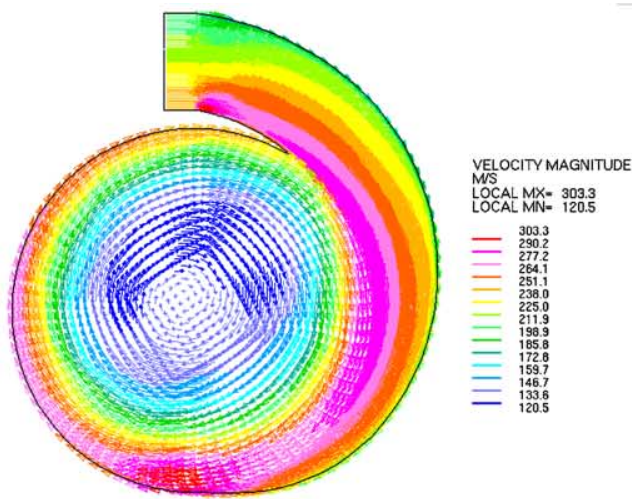

(b)

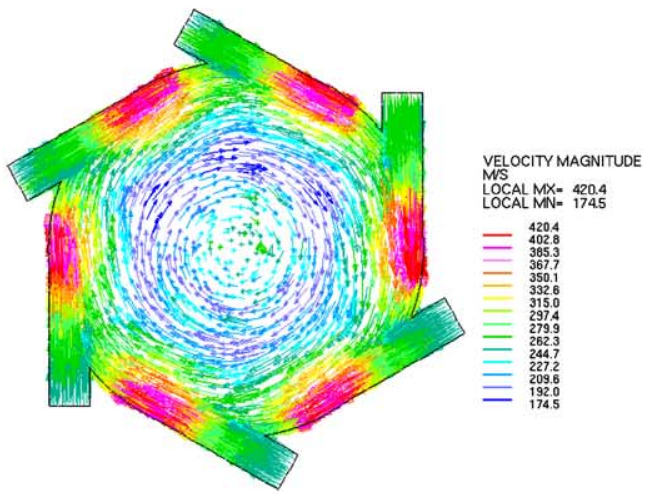

(d)

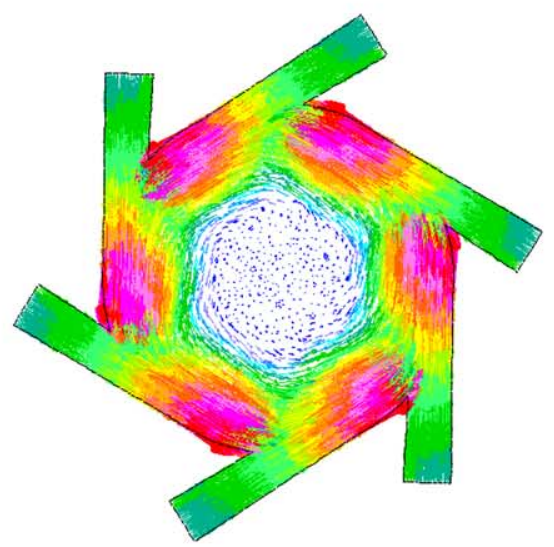

VELOCITY MAGNITUDE

VELOCITY MAGNITUDE LOCAL MX: 310.6
LOCAL MN $=120.3$

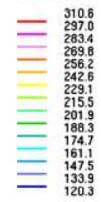

(e)

Fig. 7. Swirl patterns at the inlet zone obtained from CFD for: (a) two numbers of convergent nozzles; (b) single helical circular nozzle; (c) single helical rectangular nozzle; (d) straight six numbers of nozzles; (e) convergent six numbers of nozzles.

fractions and $L / D$ ratios for $d_{\mathrm{c}}=7 \mathrm{~mm}$ and $d_{\mathrm{c}}=6 \mathrm{~mm}$ respectively. It is seen that a hot gas fraction of $12-15 \%$ gives maximum hot gas temperature of $391 \mathrm{~K}$ for $\mathrm{L} /$ $D=30$ and for cold gas fraction $\approx 60 \%$ (lowest cold gas fraction possible with the present experimental system) gives lowest cold gas temperature of $267 \mathrm{~K}$ for $L / D=35$.

\section{Secondary circulation flow}

CFD studies were carried out to verify the existence of secondary circulation flow in vortex tube [13] and its influence on temperature separation for different $d_{\mathrm{c}} / D$ values. Analysis were carried out for 
Table 1

Comparison of nozzle exit velocity and total temperature difference between cold and hot ends for five different nozzle configurations

\begin{tabular}{lllll}
\hline Nozzle profile & No. of nozzles & Mass flow rate at inlet $(\mathrm{kg} / \mathrm{s})$ & Nozzle exit velocity $(\mathrm{m} / \mathrm{s})$ & $\begin{array}{l}\text { Total temperature difference, } \\
\Delta T_{\text {ch }}(\mathrm{K})\end{array}$ \\
\hline Convergent & 2 & 0.00890 & 362.8 & 20.2 \\
Circular helical & 1 & 0.00905 & 303.3 & 19.8 \\
Rectangular helical & 1 & 0.00892 & 310.6 & 19.6 \\
Straight & 6 & 0.00921 & 420.4 & 21.6 \\
Convergent & 6 & 0.00917 & 537.9 & 22.3 \\
\hline
\end{tabular}

$12 \mathrm{~mm}$ diameter vortex tube for $d_{\mathrm{c}} / D$ values of 0.323 , $0.417,0.5$ and 0.58 for $L / D=30$. We find that there is secondary circulation flow superimposed on the primary forced vortex for lower values of $d_{\mathrm{c}} / D$ as reported by Ahlborn et al. [13]. However we find that as $d_{\mathrm{c}} / D$ value is increased, the magnitude of secondary flow decreases and is completely eliminated for $d_{\mathrm{c}} / D=$ 0.58 (cold end diameter $=7 \mathrm{~mm}$ ). The velocity profiles of the flow near the cold end exit for $d_{\mathrm{c}} \mathrm{d} D=0.323$ and $d_{\mathrm{c}} / D=0.58$ are shown in Fig. 11(a) and (b) respectively. They clearly show the swirling back of the secondary flow near the cold end exit for vortex tube with $d_{\mathrm{c}} / D=0.323$ and absence of secondary flow for $d_{\mathrm{c}} / D=0.58$. The stream function plotting for the vortex tube of $d_{\mathrm{c}} / D=0.417$ near the cold end exit zone in Fig. 12 shows a few of the secondary flow loops as well as the free and forced vortex flow components.

We have evaluated the ratios of measured exit temperatures as a function of calculated values for $12 \mathrm{~mm}$ diameter vortex tube at different values of $d_{\mathrm{c}} / D$ based on the analytical model of Ahlborn et al. [14]. The results are shown in Fig. 13. It is observed that for lower values of $d_{\mathrm{c}} / D$, the ratios of exit temperatures are closer to the analytical model but for higher $d_{\mathrm{c}} / D$ values, the measured values are much higher than the predicted values. Therefore the analytical model by Ahlborn et al. [14] appears to be valid for the specific vortex tube with low $d_{\mathrm{c}} / D$ value of 0.323 .

The temperature separation in vortex tube with low $d_{\mathrm{c}} / D$ values was lower compared to higher $d_{\mathrm{c}} / D$ values (Figs. 8(a) and 13). It is to be noted that Ahlborn et al. [13] have observed secondary circulation flow for a vortex tube with a rather low $d_{\mathrm{c}} / D$ value of 0.323 and have not evaluated the flow for any other $d_{\mathrm{c}} / D$ value. Secondary circulation flow appears to be characteristic of vortex tubes with low $d_{\mathrm{c}} / D$ values as confirmed by our studies and could be a performance degrading mechanism. The degradation could be due to transfer of colder fluid elements near the cold end exit zone through the swirling secondary loop to the warmer flow region causing decrease in the hot end temperature and transfer of warmer flow elements back to the cold end exit zone causing increase in cold end exit temperature of the flow.

\section{Thermal analysis of the optimized vortex tube}

The performance characteristics of the vortex tubes have been estimated by calculating the coefficient of performance (COP) of this system as a heat engine and as a refrigerator. As a heat engine the $\mathrm{COP}$ of the vortex tube is given as

$(\mathrm{COP})$ he $=m_{\mathrm{h}}\left(h_{\mathrm{h}}\right) / m_{\mathrm{i}}\left(h_{\mathrm{i}}\right)=(1-\alpha)\left(h_{\mathrm{h}} / h_{\mathrm{i}}\right)$

As a refrigerator the COP of the vortex tube is given as

$$
(\mathrm{COP}) \mathrm{re}=m_{\mathrm{c}}\left(h_{\mathrm{c}}\right) / m_{\mathrm{i}}\left(h_{\mathrm{i}}\right)=\alpha\left(h_{\mathrm{c}} / h_{\mathrm{i}}\right)
$$

The COP values of the vortex tube for $L / D$ equal to 10 and 30 as a heat engine and as a refrigerator are shown in Fig. 14. It is observed that the COP of the vortex tube as a heat engine varies from 0.16 to 0.59 for $L / D=10$ and varies from 0.16 to 0.46 for $L / D=30$. The COP of this vortex tube as a refrigerator varies from 0.42 to 0.83 for $L / D=10$ and varies from 0.55 to 0.82 for $L / D=30$. This shows that $L / D$ ratio has negligible effect on COP of the vortex tube.

The COP values of Carnot cycle for the same temperature conditions as shown in Fig. 14 for $L / D$ equal to 10 varies from 10.5 to 26.5 as a refrigerator and 6.8 to 10.9 as a heat engine. For $L / D$ equal to 30 the COP values varies from 8.6 to 16.9 as a refrigerator and 4.3 to 6.1 as a heat engine.

The COP of vortex tube is very low as compared to COP of Carnot cycle. However this is a unique device which produces both heating and cooling effects simultaneously without using any other form of energy than compressed air at moderate pressure. This device can be used effectively in process environments where heating and cooling outputs of vortex tubes can be concurrently used.

\section{Conclusion}

A new approach in optimizing the design of vortex tube has been evolved through CFD analysis using $k-\varepsilon$ turbulence model of the Star-CD code, replacing experimental correlation used hitherto by other researchers. The accuracy of the CFD simulations has been validated by experiments. The swirl velocity, axial velocity and 

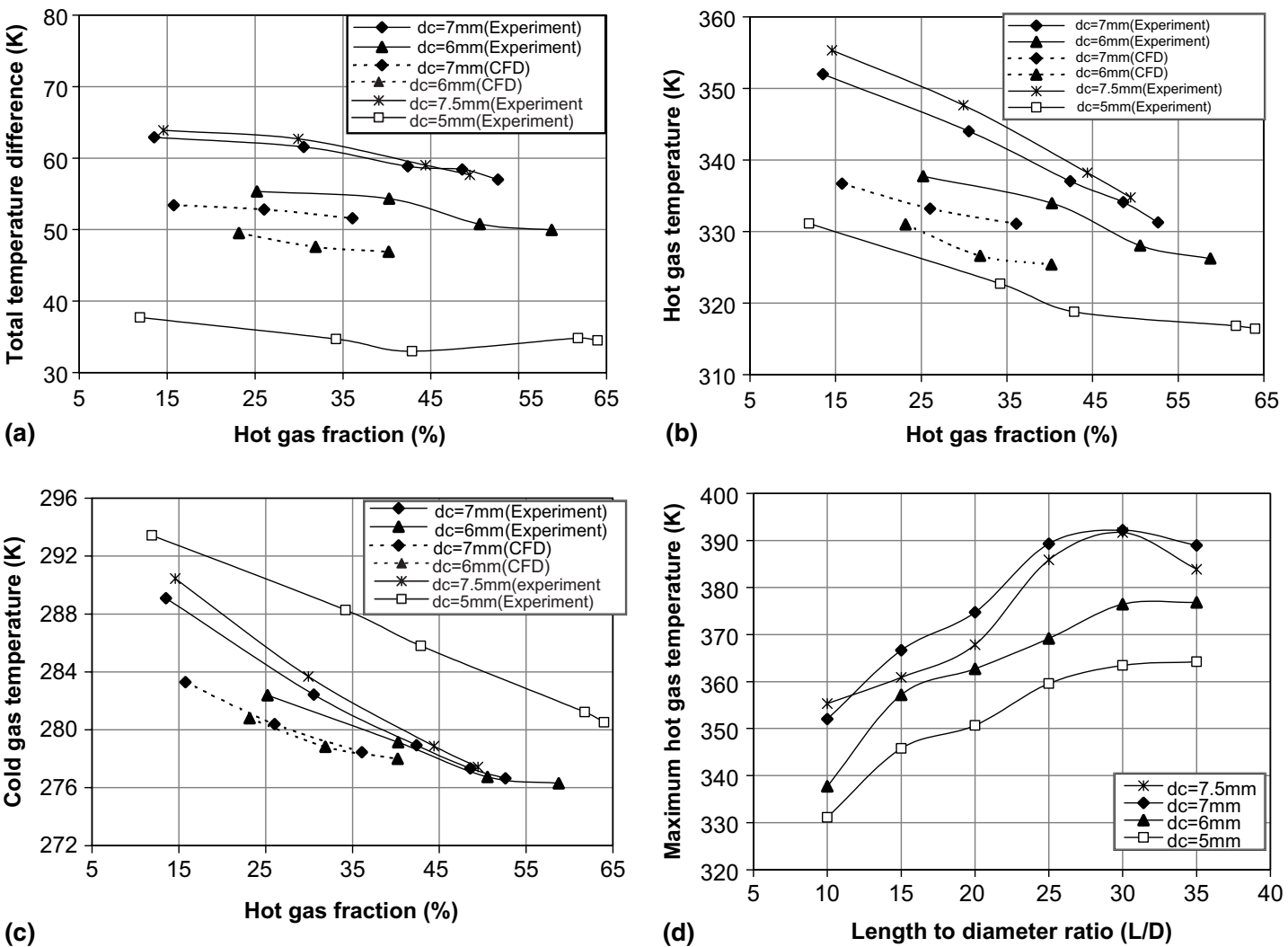

(c)

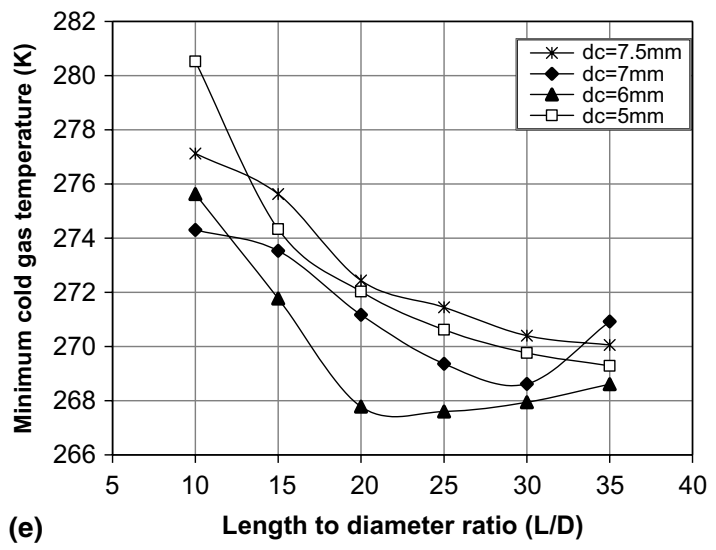

Fig. 8. (a) Total temperature difference at different hot gas fractions and cold end diameters for $L / D=10$; (b) hot gas temperature at different hot gas fractions and cold end diameters for $L / D=10$; (c) cold gas temperature at different hot gas fractions and cold end diameters for $L / D=10$; (d) experimental maximum hot gas temperature obtained at different $L / D$ ratios and cold end diameters; (e) experimental minimum cold gas temperature obtained at different $L / D$ ratios and cold end diameters.

radial velocity components of the flow and the flow pattern have been obtained through CFD, which are difficult to obtain experimentally due to disturbance of flow by measuring probes. The analysis shows that the flow has forced and free vortex components up to stagnation point and temperature difference between hot and cold gas flow can be maximized by increasing the length to diameter ratio of vortex tube such that stagnation point is farthest from the nozzle inlet and within the tube.

Many investigators have so far determined the optimum nozzle profile and number of nozzles of vortex tube by experimental investigations. The work reported here shows that CFD analysis provides an elegant and 


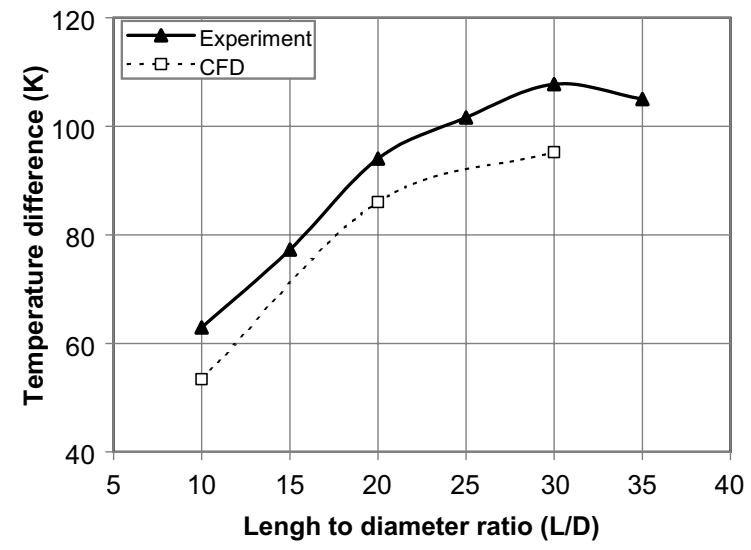

Fig. 9. Temperature difference between hot and cold gas for different $L / D$ ratios.
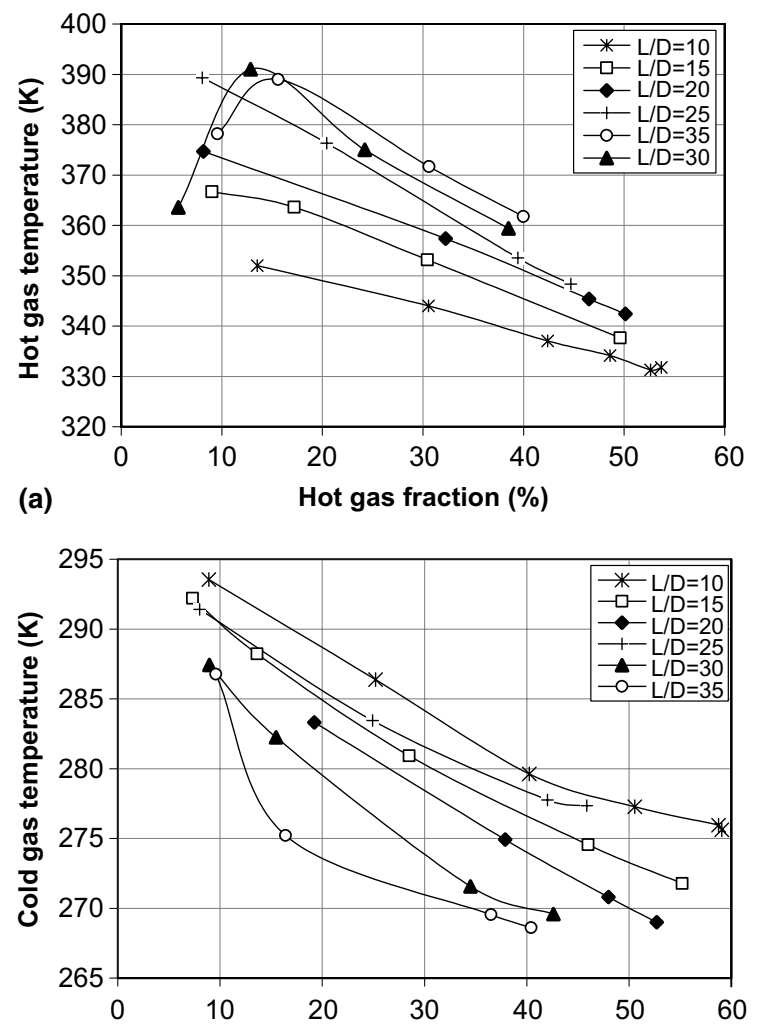

(b)

Hot gas fraction (\%)

Fig. 10. (a) Hot gas temperature at different hot gas fractions and $L / D$ ratios at $d_{\mathrm{c}}=7 \mathrm{~mm}$; (b) cold gas temperature at different hot gas fractions and $L / D$ ratios at $d_{\mathrm{c}}=6 \mathrm{~mm}$.

more accurate way to arrive at these critical parameters. For a $12 \mathrm{~mm}$ diameter vortex tube this study has shown that swirl generator with six numbers of convergent nozzles gives the best performance.

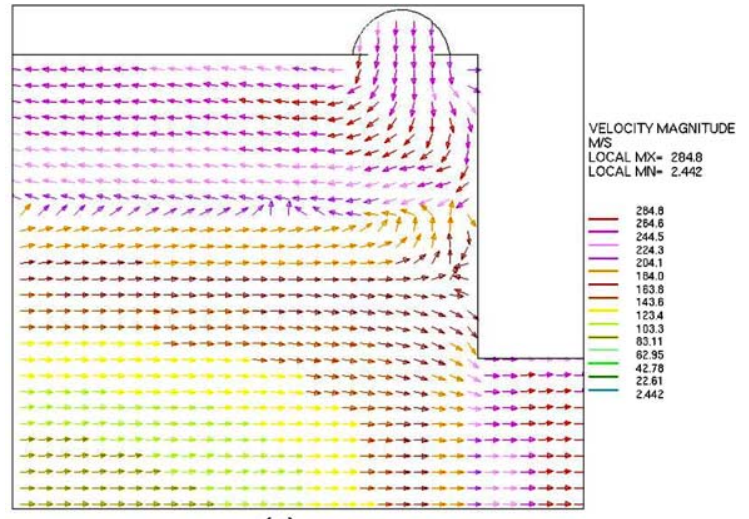

(a)

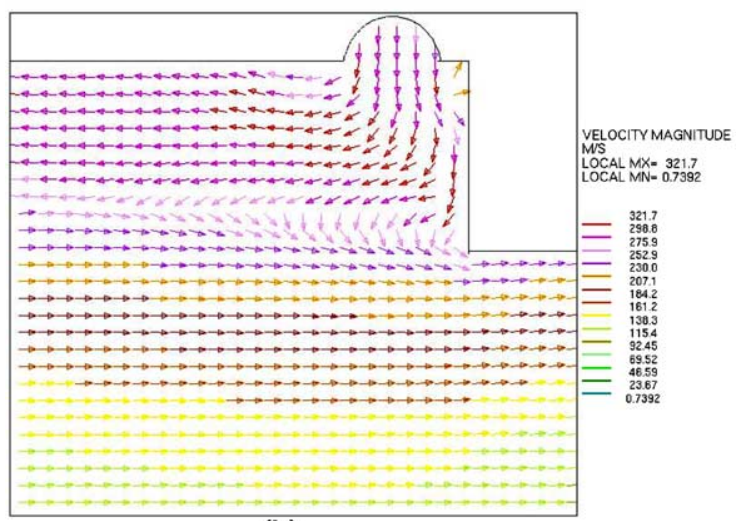

(b)

Fig. 11. (a) Flow pattern near cold end exit for $d_{\mathrm{c}} / D=0.323$ showing secondary circulation flow; (b) flow pattern near cold end exit for $d_{\mathrm{c}} / D=0.58$ showing no secondary circulation flow.

The critical design parameters of the vortex tubes, namely the cold end diameter $\left(d_{\mathrm{c}}\right)$ and the length to diameter ratio $(L / D)$ also could be most effectively decided by CFD techniques rather than depending on experimental correlations, which are conventionally used by vortex tube designers. The accuracy of these simulated results for $12 \mathrm{~mm}$ diameter tube have been validated by experimental investigations.

The CFD and experimental studies have shown that for $12 \mathrm{~mm}$ diameter vortex tube, the cold end diameter of $7 \mathrm{~mm}$ is ideal for producing maximum hot gas temperature, while cold end diameter of $6 \mathrm{~mm}$ is optimum for reaching the minimum cold gas temperature. The investigations have shown that $L / D$ ratio in the range of 25-35 is optimum for achieving best thermal performance for $12 \mathrm{~mm}$ vortex tube. These optimized vortex tube could produce maximum hot gas temperature of $391 \mathrm{~K}$ at $12-15 \%$ hot gas fraction and a minimum cold gas temperature of $267 \mathrm{~K}$ at about $60 \%$ cold gas fraction. 


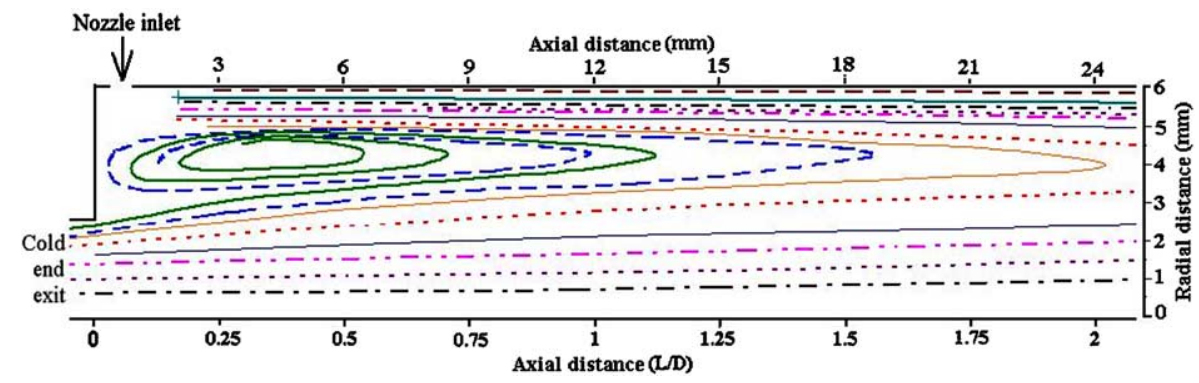

Fig. 12. Stream function for $d_{\mathrm{c}} / D=0.417$ near cold end exit zone.

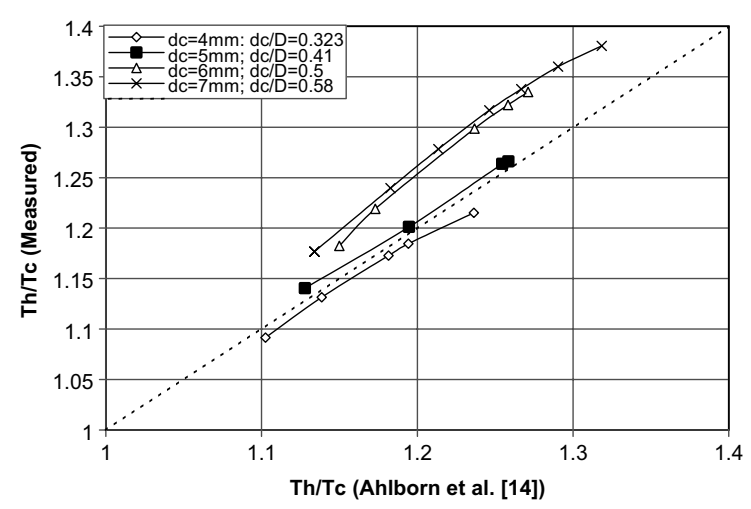

Fig. 13. Ratio of measured exit temperatures as function of calculated values based on analytical model of Ahlborn et al. [14] for different cold end diameters.

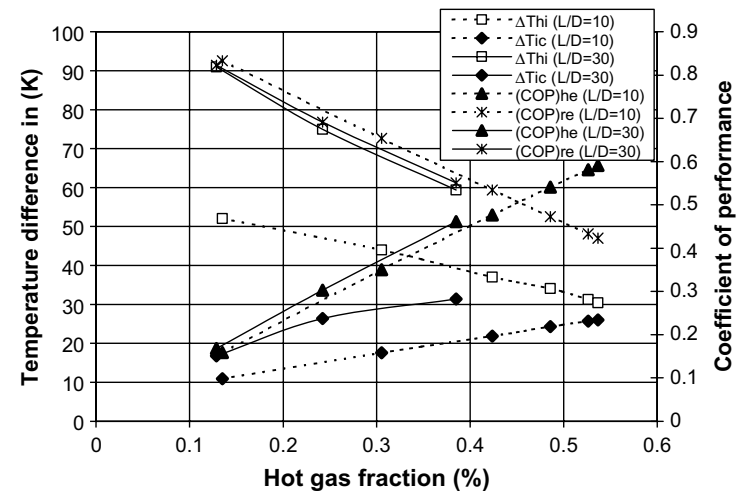

Fig. 14. Coefficient of performance of the optimized vortex tube as a heat engine and as a refrigerator for $L / D=10$ and $L / D=30$ at inlet pressure of $0.5422 \mathrm{MPa}$ (absolute) and inlet temperature of $300 \mathrm{~K}$.

The studies conducted by us have confirmed the presence of secondary flow for vortex tubes with low $d_{\mathrm{c}} / D$ values. However CFD analysis and experimental results indicate that secondary circulation flow could be a performance degrading mechanism in vortex tubes. An optimally designed vortex tube has only two regimes of forced and free vortex flows and with optimal $d_{\mathrm{c}} / D$ value, the secondary flow is eliminated which results in its higher temperature separation between cold end and hot end. For the $12 \mathrm{~mm}$ vortex tube the optimum $d_{\mathrm{c}}$ value is $7 \mathrm{~mm}$ corresponding to $d_{\mathrm{c}} / D=0.58$.

The maximum COP of the vortex tube is found to be 0.59 as a heat engine and 0.83 as a refrigerator. Though the COP of vortex tube is low as compared to the COP of Carnot cycle for the same temperature conditions, the thermal separation produced by the vortex tubes can find ready applications where there are modest heating and/or cooling requirements and ready availability of compressed air.

\section{References}

[1] G.J. Ranque, Experiences sur la detente giratoire avec productions simultanes d'un echappement d'air chaud et d'un echappement d'air froid, J. Phys. Radium 4 (7) (1933) $112-114$

[2] R. Hilsch, The use of the expansion of gases in a centrifugal field as cooling process, Rev. Sci. Instrum. 18 (2) (1947) 108-113.

[3] R.G. Deissler, M. Perlmutter, Analysis of the flow and energy separation in a turbulent vortex, Int. J. Heat Mass Transfer 1 (1960) 173-191.

[4] M. Kurosaka, Acoustic streaming in swirl flow and the Ranque-Hilsch (vortex-tube) effect, J. Fluid Mech. 124 (1982) 139-172.

[5] B. Ahlborn, J.U. Keller, R. Staudt, G. Treitz, E. Rebhan, Limits of temperature separation in a vortex tube, Phys. D: Appl. Phys. 27 (1994) 480-488.

[6] T.T. Cockerill, Thermodynamics and Fluid Mechanics of a Ranque Hilsch Vortex Tube, Masters Thesis, University of Cambridge, England, 1995.

[7] A.F. Gutsol, The Ranque effect, Phys.-Espekhi 40 (6) (1997) 639-658.

[8] A.P. Merkulov, Vikhrevoi Effekt I Ego Primenenie V Tekhnike (Vortex Effect and Its Application in Technique), Mashinostroenie, Moscow, 1969.

[9] V.I. Metenin, Investigation of vortex temperature type compressed gas separators Translated from, Z. Tekh. Fiz. 30 (9) (1960) 1095-1103.

[10] W. Frohlingsdorf, H. Unger, Numerical investigations of the compressible flow and the energy separation in 
Ranque-Hilsch vortex tube, Int. J. Heat Mass Transfer 42 (1999) 415-422.

[11] Star-CD, Version 3.10A: Methodology and User Guide, Computational Dynamics Limited, UK, 1999.

[12] H. Takahama, Studies on vortex tubes, Bull. JSME 8 (31) (1965) 433-440.
[13] B. Ahlborn, S. Groves, Secondary flow in vortex tube, Fluid Dyn. Res. 21 (1997) 73-86.

[14] B.K. Ahlborn, J.U. Keller, E. Rebhan, The heat pump in a vortex tube, J. Non-Equilib. Thermodyn. 23 (2) (1998) 159-165. 